\title{
Immunosuppressive Therapeutic Complications in a Lung Transplant Patient
}

\author{
Sofia Moreira-Silva ${ }^{\mathrm{a}, \mathrm{e}}$, Catarina Pereira ${ }^{\mathrm{a}}$, Joana Urbano ${ }^{\mathrm{a}}$, Joana Pimenta $^{\mathrm{a}, \mathrm{b}}$, Carla Damas $^{\mathrm{c}}$, \\ Paula Dias ${ }^{\mathrm{a}, \mathrm{d}}$, Fernando Frioes ${ }^{\mathrm{a}, \mathrm{b}, \mathrm{d}}$, Jorge Almeida ${ }^{\mathrm{a}, \mathrm{d}}$
}

\begin{abstract}
The number of lung transplants has increased in the last years. Besides, the complications of immunosuppressive treatment, such as infections, have also been raising. Another complication, although seldom described, is thrombotic microangiopathy associated with calcineurin inhibitors. We report a case of a 65 -year-old woman with a 6-month bi-pulmonary transplant, admitted for pneumonia. Addressing the differential diagnosis, pneumonia in immunosuppressed patient, aspergillosis, CMV disease and acute rejection were considered. High levels of tacrolimus were identified. The patient evolved with multiple organ dysfunctions. Hemodialysis was started on the third day. On the 5th day, patient developed progressive anemia, leukopenia and thrombocytopenia, schistocytes were present on peripheral smear and Coombs test was negative. A thrombotic microangiopathy probably related to tacrolimus high levels was identified. Plasma exchange was initiated on the eighth day, and intensified on the 11th day, without success, and on 14th day, the patient died. We believe our case report is relevant, since thrombotic microangiopathy in lung transplant has rarely been described and lung transplant is a growing reality, as well as its complications. Furthermore, our case is more severe than the ones described in literature, possibly due to prolonged tacrolimus toxic levels and concomitant infection.
\end{abstract}

Keywords: Lung transplant; Immunosuppressive agents; Immunosuppressive therapeutics; Thrombotic microangiopathy; Calcineurin inhibitors; Hemolytic uremic syndrome

Manuscript accepted for publication November 19, 2015

aDepartment of Internal Medicine, Sao Joao Hospital Centre, Porto, Portugal ${ }^{b}$ Department of Medicine \& Unit of Cardiovascular Research \& Development, Faculty of Medicine, University of Porto, Porto, Portugal

'Department of Pneumology, Sao Joao Hospital Centre, Porto, Portugal

dUnidade Cuidados Intermedios de Medicina, Sao Joao Hospital Centre, Porto, Portugal

${ }^{\mathrm{e} C o r r e s p o n d i n g ~ A u t h o r: ~ S o f i a ~ M o r e i r a ~ d a ~ S i l v a, ~ S e r v i c o ~ d e ~ M e d i c i n a ~ I n t e r n a, ~}$ Centro Hospitalar de Sao Joao, Alameda Prof. Hernani Monteiro, 4200-319, Porto, Portugal. Email: siamms@gmail.com

doi: http://dx.doi.org/10.14740/jmc2373w

\section{Introduction}

Substantial progress has been made in immunosuppressive regimens to prevent acute and chronic rejection in lung transplant, while trying to reduce the side effects [1]. Infectious complications remain a leading cause of death after lung transplantation, accounting for $35 \%$ of deaths in the first year [2]. The calcineurin inhibitors $(\mathrm{CI})$ are the mainstay of immunosuppression in lung transplant. Thrombotic microangiopathy (TMA) is a complication that has been increasingly reported in the recent years $[3,4]$ and CI have been implicated in most cases [5]. We report a case of TMA in a lung transplant recipient receiving tacrolimus, with concomitant infection.

\section{Case Report}

A 65-year-old Caucasian woman had received a bi-pulmonary transplant 6 months before the recent admission. The postoperative period was complicated with acute lung rejection, Pseudomonas aeruginosa pneumonia and pulmonary thromboembolism (treated with high dose glucocorticoids, antibiotics and anticoagulation). She developed chronic renal failure in relation to CI nephrotoxicity. Her maintenance immunosuppression included tacrolimus, mycophenolate mofetil and prednisolone. Her prophylaxis included inhaled amphotericin B lipid complex, colistimethate and sulfamethoxazole/trimethoprim. In March 2014, she was admitted for gastroenteritis and treated with ciprofloxacin and fluids. No lesions were found in endoscopic study and colonic biopsies were unremarkable. The CMV load blood assay revealed 36,000 copies/mL. Valganciclovir was added and mycophenolate mofetil was switched to mycophenolic acid with clinical improvement. In a follow-up visit in May 2014, spirometry showed decline in lung volumes and a bronchofibroscopy was performed. The patient missed next visits and stopped anticoagulation on her own. On July 2014, she was admitted to the hospital with dyspnea, cough, sputum and malaise for the last 2 weeks. On examination, she was tachypneic, and had BP of 105/63 mm Hg and HR of $97 \mathrm{bpm}$; lung auscultation showed crackles on the lower half of the right lung and rhonchi bilaterally. The remaining physical examination was unremarkable. Blood gas analysis showed hypoxemia with $\mathrm{PaO}_{2} / \mathrm{FiO}_{2}$ ratio 210 and chest XRay showed a right lung hypodensity (Fig. 1). Blood sample 


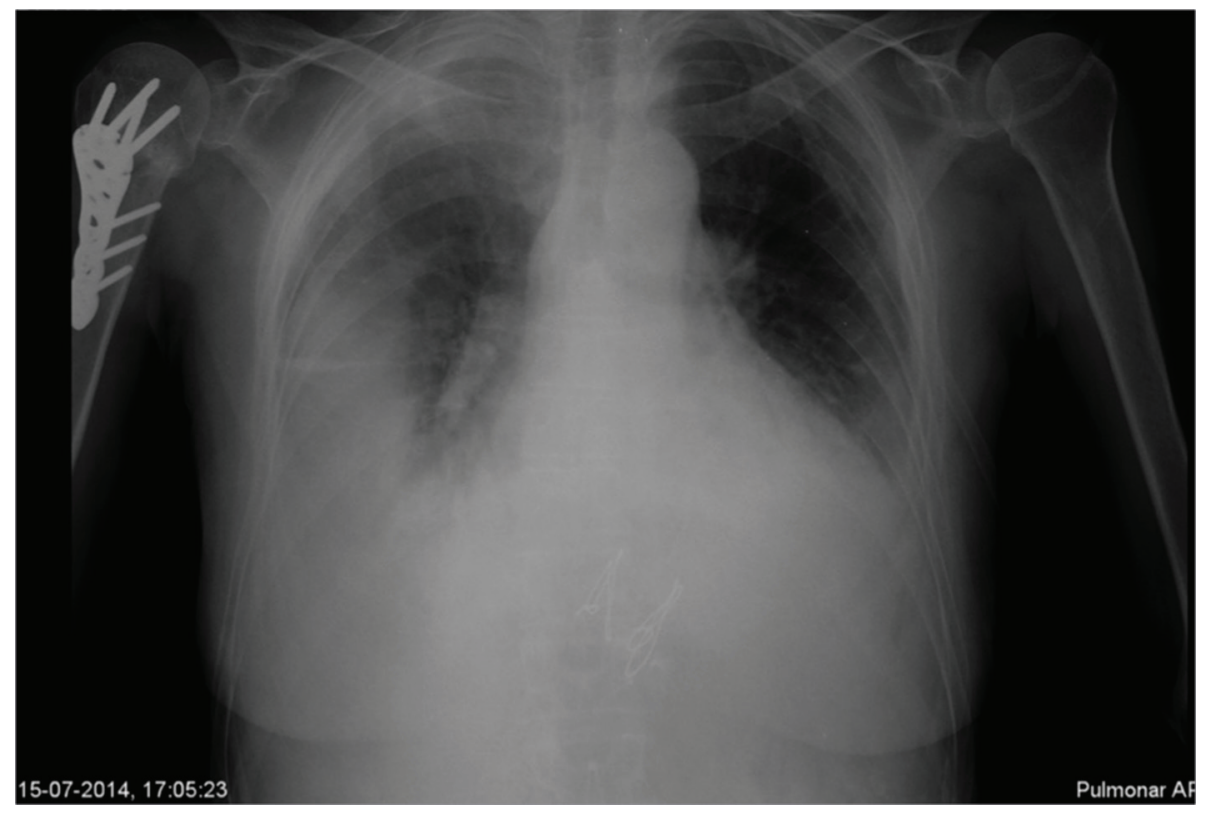

Figure 1. Chest X-ray showing right hypodensity.

analysis revealed $\mathrm{Hb} 11.5 \mathrm{~g} / \mathrm{dL}$, leucocytes $10 \times 10^{9} / \mathrm{L}, 9.2 \times$ $10^{9} / \mathrm{L}$ neutrophils, $0.3 \times 10^{9} / \mathrm{L}$ lymphocytes, $249 \times 10^{9} / \mathrm{L}$ platelets, urea/creatinine 126/2.59 mg/dL, Na $126 \mathrm{mEq} / \mathrm{L}, \mathrm{K}^{+} 5.6$ $\mathrm{mEq} / \mathrm{L}$, lactate dehydrogenase $338 \mathrm{U} / \mathrm{L}$, and C-reactive protein $176 \mathrm{mg} / \mathrm{L}$. A diagnosis of pneumonia was made. Reviewing the patient previous medical records, on routine bronchial lavage performed on May, Aspergillus fumigatus was identified on mycologic sample and the polymerase chain reaction (PCR) for A. fumigatus and CMV were positive. Transbronchial biopsy was not suggestive of rejection. She was admitted to intensive care unit and treated with piperacillin/tazobactam and voriconazol. The mycophenolic acid was suspended. A thoracic tomography scan (CT) revealed moderate loculated pleural effusion (Fig. 2), and several areas of consolidation. Thoracentesis was performed and an exudative pleural fluid, rich in neutrophils with $\mathrm{pH} 7.3$ was drained. On the second day, tacrolimus level was $68 \mathrm{ng} / \mathrm{mL}$ (range $5-20 \mathrm{mg} / \mathrm{dL}$ ), probably in relation to interaction with voriconazol, and it was suspended. In the next days, the patient evolved with multiple organ dysfunctions: neurologic (normal brain CT), respiratory

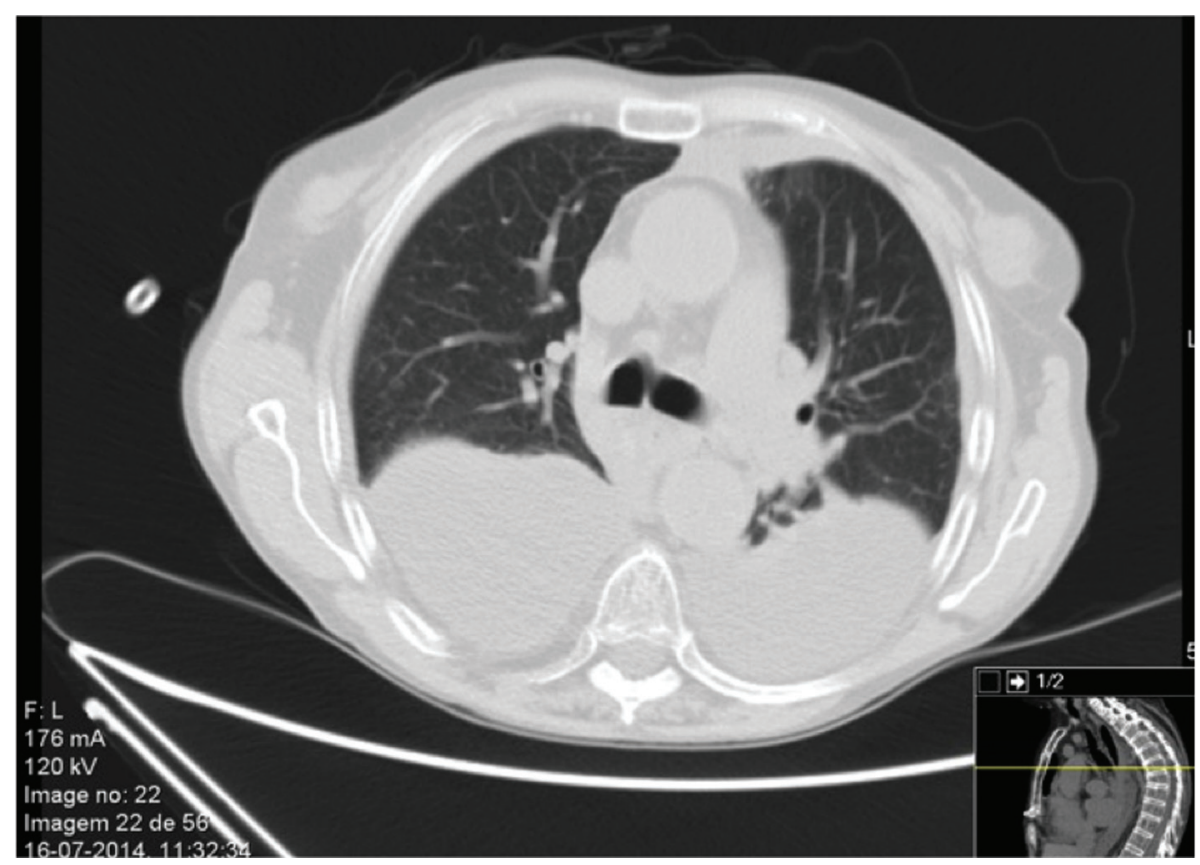

Figure 2. Thoracic tomography scan revealing a moderate volume pleural effusion. 
(non-invasive positive pressure ventilation was started) and electrical, with two episodes of atrial fibrillation. Due to clinical deterioration, high dose intravenous methylprednisolone was started for a possible acute rejection beyond infection. Renal failure progressed and patient started hemodialysis on the third day. At that moment, all the blood cultures were negative as well as the mycologic and bacteriologic cultures of pleural effusion. The PCR assay to A. fumigatus was negative but the PCR for CMV was positive in different pleural fluid samples. Blood CMV PCR showed 42,000 copies/mL. Ganciclovir was associated.

On the fifth day, patient developed progressive anemia, leukopenia and thrombocytopenia, schistocytes were present on peripheral smear and Coombs test was negative. A TMA, probably related to tacrolimus high levels, was identified and plasma exchange was initiated on the eighth day. Tacrolimus levels remained over therapeutic range until the ninth day. On the 11th day, due to unresponsiveness, plasma exchange was intensified to twice daily, without success, and on the 14th day, the patient died.

\section{Discussion}

The two main complications of lung transplant are rejection and infection. Differential diagnosis can be difficult, particularly in the cases when both entities coexist. This patient was initially treated for pneumonia and later, received high dose corticosteroids to a possible acute rejection. When the immunophenotyping of pleural effusion became available, it was not suggestive of rejection and the patient had no conditions for transbronchial biopsy.

At 6 months of transplant, opportunistic pathogens, as well as community and hospital acquired organisms have to be considered. In our patient, microbiologic results prior to admission suggested pulmonary aspergillosis and so voriconazol was started.

The CIs are essential components of maintenance immunosuppression following lung transplantation. They are metabolized by cytochrome P450 3A and a variety of drug interactions can occur, namely with voriconazol, increasing the tacrolimus levels [6]. There may be multiple mechanisms for toxic drug-mediated kidney injury. The CI (such as tacrolimus) can cause endothelial dysfunction and increased platelet aggregation possibly through the inhibition of prostacyclin. They also inhibit the VEGF function in renal endothelial cells and podocytes and cause gradual development of glomerular TMA $[7,8]$. This toxicity seems to be dose- and time-dependent $[7$, 9].

Tacrolimus-associated TMA is a rare, but potentially fatal, complication in solid organ transplantation, with an estimated incidence of $1-4 \%[5,10]$. In lung transplant, the estimated incidence is $2.3 \%$, but only four case series and 11 cases have been reported $[3,4,8]$. Fifty percent of cases occurred during concurrent disease as in our report [3]. In a recent review, a survival rate of $38.9 \%$ was described [3]; however, in the majority of case reports, TMA is associated with a favorable prognosis and managed with suspension or only dose reduc- tion of CI $[4,10,11]$. In our case, tacrolimus was stopped, plasma exchange was started and intensified but still without success. Prolonged toxic levels and severe concurrent disease may had been contributing factors for the adverse outcome in our patient.

\section{Conclusions}

As lung transplant is a growing reality, physicians should be alert to potential complications. TMA is a rare complication of CI, which in our case was more severe than the previously described in literature. Prolonged tacrolimus toxic levels and concomitant infection might have contributed.

\section{Conflict of Interest}

None.

\section{References}

1. Yusen RD, Christie JD, Edwards LB, Kucheryavaya AY, Benden C, Dipchand AI, Dobbels F, et al. The Registry of the International Society for Heart and Lung Transplantation: Thirtieth Adult Lung and Heart-Lung Transplant Report--2013; focus theme: age. J Heart Lung Transplant. 2013;32(10):965-978.

2. Trulock EP, Edwards LB, Taylor DO, Boucek MM, Keck BM, Hertz MI. Registry of the International Society for Heart and Lung Transplantation: twenty-second official adult lung and heart-lung transplant report--2005. J Heart Lung Transplant. 2005;24(8):956-967.

3. Verbiest A, Pirenne J, Dierickx D. De novo thrombotic microangiopathy after non-renal solid organ transplantation. Blood Rev. 2014;28(6):269-279.

4. Reig Mezquida JP, Jover AS, Ansotegui Barrera E, Escriva Peiro J, Pastor Colom MD, Pastor Guillem J. Thrombotic microangiopathy associated with tacrolimus in lung transplantation. Arch Bronconeumol. 2015;51(5):e23-24.

5. Nwaba A, MacQuillan G, Adams LA, Garas G, Delriviere L, Augustson B, DeBoer B, et al. Tacrolimus-induced thrombotic microangiopathy in orthotopic liver transplant patients: case series of four patients. Intern Med J. 2013;43(3):328-333.

6. Dodds-Ashley E. Management of drug and food interactions with azole antifungal agents in transplant recipients. Pharmacotherapy. 2010;30(8):842-854.

7. George JN, Nester CM. Syndromes of thrombotic microangiopathy. N Engl J Med. 2014;371(19):1847-1848.

8. Sartelet H, Toupance O, Lorenzato M, Fadel F, Noel LH, Lagonotte E, Birembaut P, et al. Sirolimus-induced thrombotic microangiopathy is associated with decreased expression of vascular endothelial growth factor in kidneys. Am J Transplant. 2005;5(10):2441-2447.

9. Katari SR, Magnone M, Shapiro R, Jordan M, Scantlebury V, Vivas C, Gritsch A, et al. Clinical features of acute reversible tacrolimus (FK 506) nephrotoxicity in kidney 
transplant recipients. Clin Transplant. 1997;11(3):237242.

10. Shitrit D, Starobin D, Aravot D, Fink G, Izbicki G, Kramer M. Tacrolimus-induced hemolytic uremic syndrome case presentation in a lung transplant recipient. Transplant Proc. 2003;35(2):627-628.

11. Radhi M, Carpenter SL. Thrombotic microangiopathies. ISRN Hematol. 2012;2012:310596. 